Herz 2011 · 36:175-176

DOI 10.1007/s00059-011-3456-1

Online publiziert: 21. April 2011

(c) Urban \& Vogel 2011

\author{
R. Erbel \\ Westdeutsches Herzzentrum Essen, Klinik für Kardiologie, \\ Universitätsklinikum Essen, Essen
}

\title{
Aktuelle interventionelle koronare Intervention
}

Die Diagnostik und Therapie der koronaren Herzerkrankung hat wesentliche Fortschritte erzielt. Zum Teil ganz unbemerkt von der breiten Öffentlichkeit sind auch für die katheterbasierte perkutane koronare Intervention (PCI) wichtige Neuerungen eingeführt worden. War noch vor wenigen Jahren die Nutzung der medikamentenbeschichteten Stents (DES) umstritten, gehören sie heute zum Alltag in jedem Katheterlabor. In diesem Heft stellen M. Kollum und Ch. Bode die aktuellen Entwicklungen vor und beleuchten die Fortschritte. Die Vorteile und Sicherheit der 2. und 3. DESGeneration werden von I. Akin und Mitarbeitern aus der Arbeitsgruppe von Ch. Nienaber vertieft analysiert. Die Vorteile liegen in neuen verwendeten Materialien wie den Kobalt-Chrom- und Platin-Chrom-Legierungen, vor allen Dingen aber auch in der Nutzung neuer Polymere, die sich im Verlaufe von wenigen Wochen auflösen, nachdem das antiproliferative Medikament des DES abgegeben worden ist und ein reiner Metall-Stent („bare metal stent“, BMS) zurückbleibt.

Außerdem sind Substanzen, vorwiegend Analoge zum Sirolimus, wie Everolimus und Biolimus A9, nach experimenteller Austestung für die DES-Beschichtung eingesetzt worden.

Von großem innovativem Potenzial sind auch neue Stents, die in den StentStreben vorpräparierte Einkerbungen enthalten und die Polymerbeschichtung auf die Stellen begrenzen, die mit Medikamenten aufgefüllt werden. Interessant ist dabei der Aspekt, dass auch mehrere Substanzen, unterschiedlich verteilt, auf einen solchen Stent aufgebracht werden könnten. Durch diese Technik wird die Medikamentenabgabe stark konzentriert und auf der anderen Seite die Polymerbeschichtung weiter begrenzt.

Sicherlich ist ein Fortschritt auch darin zu sehen, dass bei Polymerbeschichtung nur die Seite des Stents, die zur Gefäßwand ausgerichtet ist, eine Beschichtung trägt.

In den letzten Jahren ist zusätzlich, besonders aus Deutschland kommend, die Nutzung polymerfreier Stents vorangetrieben worden, deren Oberfläche, mikroporös gestaltet, Medikamente aufnehmen kann. Positive Ergebnisse beruhen sicherlich z. T. auf der Vermeidung polymerinduzierter Reaktionen der Gefäßwand.

Ganz neu ist die Einführung von Stents, die sich im Laufe von Monaten bis 2 Jahren auflösen und mit Medikamenten beschichtet sind. Nach erfolgreicher Vortestung hat ein erster solcher PolymerStent bereits die Zulassung erhalten, was auch für den Magnesium-Stent erwartet wird, der nicht Everolimus, sondern Paclitaxel trägt und dessen klinische Testung derzeit läuft.

Bifurkationsstenosen stellen nach wie vor eine besondere Herausforderung für die interventionelle Kardiologie dar. Eine spezielle Arbeitsgruppe kümmert sich um die wissenschaftliche Bearbeitung, aber auch klinisch-technische Detailarbeit. I. Iakovou und Mitarbeiter aus der Arbeitsgruppe von C. di Mario, London, stellen die neuen Strategien vor und illustrieren die pathophysiologischen/strömungsdynamischen Aspekte.
Gleichermaßen ist die Hauptstammintervention eine besondere Herausforderung. E. Boudriot und G. Schuler stellen die Möglichkeiten und Grenzen vor. In besonderen Fällen eröffnet sich die Möglichkeit, eine operative Revaskularisierung zu umgehen. Diesbezügliche Voraussetzungen werden im Detail beschrieben. Dabei handelt es sich um eine Strategie, die die Autoren aus der SYNTAX-Studie ableiten.

Die höchsten Anforderungen, besonders im Bezug auf die investierte Zeit, stellt die Behandlung von chronischen Koronararterienverschlüssen dar. G.S. Werner aus Darmstadt beleuchtet den aktuellen Stand und die Perspektiven der Behandlung des chronischen Koronararterienverschlusses ausführlich, geht auf technische Details ein und gibt praktische Tipps, die er mit einem klinischen Beispiel untermauert - vielleicht für manche Leser eine Hilfestellung bei dieser sehr schwierigen Technik.

Die deutsche Kardiologie kann stolz darauf sein, dass mit der Entwicklung der medikamentenbeschichteten Ballons (DEB) eine Erweiterung der interventionellen Möglichkeiten für die Behandlung der koronaren Gefäßstenosen und Restenosen eröffnet wurde. B. Scheller stellt die Möglichkeiten und Grenzen der medikamentenbeschichteten Ballonkatheter vor und beleuchtet die bereits klaren Indikationen wie z. B. die Behandlung der InStent-Restenose. Aber auch weitere mögliche Anwendungsbeispiele werden gegeben und die entsprechenden klinischen Daten erörtert. Zwischenzeitlich haben sich viele Firmen dieses Themas ange- 
nommen und entsprechende Katheter entwickelt, die aber z. T. erst in größeren randomisierten Studien erprobt werden müssen und damit für die Anwender erhebliche Probleme aufwerfen, wenn die geeigneten Systeme für die Arbeit ausgesucht werden sollen. In einer Tabelle ist eine vollständige Übersicht über die derzeitigen Systeme aufgelistet.

Auch 2011 ist das Problem der akuten, subakuten oder auch späten und sehr späten Stent-Thrombose noch nicht ausgeräumt und bedarf einer intensiven Diskussion, wenn Fortschritte dargestellt, aber auch Limitationen beleuchtet werden. S. Cook und M. Oberhänsli aus Fribourg/Schweiz, stellen die aktuellen Daten vor, zeigen Möglichkeiten zur Prävention auf und beleuchten Risiken und prädisponierende Faktoren. Dabei stellen sie den Einsatz der Thrombozytenaggregationshemmer, die angemessen und konstant notwendig sind, heraus. Zum Schluss fassen sie wie folgt zusammen: „Die Stent-Thrombose sollte durch angemessene und konstante Thrombozyteninhibition sowie durch den Einsatz der DES der neueren Generation und durch optimale prozedurale Resultate zu einem seltenen Ereignis werden. “ Hiermit weisen die Kollegen auf die Notwendigkeit auch der entsprechenden optimierten Vorgehensweise bei der Stent-Implantation hin, die eine Voraussetzung ist, um Stent-Thrombosen zu vermeiden.

DES sind erheblich teurer als BMS, sind aber wirkungsstärker und reduzieren die Zahl der Revaskularisationen erheblich. Damit ergibt sich natürlich die Frage, ob sie kostengünstiger sind. F. Müller-Riemenschneider, T. Reinhold und S.N. Willich beleuchten, ob die DES der 2. Generation günstiger sind als die der 1. Generation. Die Autoren weisen auf die positiven und negativen Aspekte der 2. Generation der DES hin. Wie häufig hinkt die Kosten-Nutzen-Analyse der Einführung solcher neuer Entwicklungen hinterher, da zunächst Langzeitstudien abgewartet werden müssen und vor allen Dingen auch mehrere vergleichende Studien vorliegen müssen, bevor entscheidende Antworten gegeben werden können. Entsprechende Hinweise gibt diese Arbeit.
Die Herausgeber hoffen sehr, dass Ihnen dieses Heft gefällt und Sie manchen nützlichen Tipp erhalten und vielleicht sogar die Berücksichtigung mancher Aspekte in diesen Arbeiten die Sicherheit Ihrer Patienten erhöht. In einem nächsten Heft werden wir nach der Beleuchtung neuer therapeutischer Verfahren auch neue diagnostische Aspekte der Herzkatheteruntersuchung vorstellen.

Wir wünschen viel Freude an diesem Heft!

Prof. Dr. Raimund Erbel

für die Herausgeber

\section{Korrespondenzadresse}

Prof. Dr. med. Raimund Erbel Klinik für Kardiologie Westdeutsches Herzzentrum Essen Universitätsklinikum Essen Hufelandstr. 55, 45122 Essen erbel@uk-essen.de 